Gefässchirurgie 2013 · 18:518-519

DOI 10.1007/s00772-013-1228-3

Online publiziert: 27. September 2013

(c) Springer-Verlag Berlin Heidelberg 2013
J. Teßarek ${ }^{1}$. G. Rümenapf ${ }^{2}$

${ }^{1}$ St. Bonifatius Hospital Lingen gGmbH, Lingen

${ }^{2}$ Diakonissen-Stiftungs-Krankenhaus Speyer

\title{
Müssen wir jetzt umdenken?
}

Sehr geehrte Kolleginnen und Kollegen,

in den vergangenen 10 Jahren haben wir eine rasante Entwicklung der endovaskulären Therapieoptionen erlebt, begleitet durch die allgegenwärtige Diskussion über die Effektivität und die Dauerhaftigkeit der immer neuen sowie schnell wechselnden Verfahren im Vergleich zum gefäßchirurgischen Goldstandard. Dies geschah nicht immer wissenschaftlich, sondern häufig politisch und emotional.

Die im TASC-II-Dokument von 2007 [1] von einer interdisziplinären Arbeitsgruppe ausgesprochenen gefäßtherapeutischen Empfehlungen, die auf dem Schweregrad der arteriellen Verschlussprozesse und den Ergebnissen der damaligen Literatur beruhten, wurden schnell durch aktuellere Studiendaten und „endovaskuläre Aktivisten" infrage gestellt.

Aktuell erleben wir eine „Hype“-ähnliche Entwicklung mit aggressiver Propaganda für die „Drug-eluting“-Technologien (DET) in Form des „drug eluting balloon“ (DEB), der in manchen Vorträgen oder Veröffentlichungen bereits als die "aktuell gültige Therapieform“ bezeichnet wird, ohne dass hierfür vergleichende Studien von DEB, ,drug eluting stents“ (DES) oder Standardverfahren (Bypass) vorliegen.

Die DET hat in der Behandlung der koronaren Herzkrankheit zwar eine Kostensteigerung, dafür aber auch eine Verbesserung der Therapieergebnisse gebracht. Allerdings wurden die anfänglich als Durchbruch gefeierten Erfolge der koronaren DES durch akute Spätthrombosen mit fatalem Outcome relativiert. Die nichterfolgte oder inkomplette Reintimalisierung der Implantate resultierte in dauerhaft freiliegenden Stent-Streben, die bei Unterbrechung der dualen thrombozytenhemmen- den Therapie der Ausgangspunkt für akute Thrombosen sein können.

Mit dem Ziel, keine thrombogenen Materialien im Gefäßbett zu belassen, wurden die DEB und auch „biodegradable“ DES forciert entwickelt und eingesetzt. Die Nutzung von Ballons und degradablen oder resorbierbaren Implantaten als Träger- und Applikationssystem für die antiproliferativen Medikamente hat in den bisher veröffentlichten Studien ebenso vielversprechende Ergebnisse mit statistisch signifikanten Unterschieden hinsichtlich der Endpunkte „late lumen loss" (LLL) der Restenose- und Reinterventionsrate („target lesion revascularization", TLR) gezeigt wie die DES.

Es stellt sich aber die Frage, inwieweit die guten Ergebnisse auf die peripheren Gefäßbereiche übertragen werden können. Im Koronargefäß ist die Restenose das entscheidende Kriterium für den klinischen Erfolg der Primärtherapie, und sie bestimmt das Risiko eines kardial ischämischen Ereignisses. Der Endpunkt „LLL“ als Ausdruck der effektiv inhibierten intimalen Hyperplasie bzw. der effektiven Behandlung einer Stenose hat eine weit höhere Bedeutung als im peripheren Gefäßbereich. Bisher wurde die Reverschlussrate als Zeichen der Effektivität einer Therapie (Beinerhalt) seitens der Interventionalisten vehement in ihrer Relevanz bestritten. Beinerhalt und nicht Offenheitsrate war der ultimative Endpunkt aller Studien bei Patienten mit chronischkritischer Extremitätenischämie.

Deshalb ist es erstaunlich, dass von endovaskulären Therapeuten jetzt plötzlich "LLL" und „Offenheitsrate“ als wesentliche Kriterien für die Effektivität ihrer Therapieform angeführt werden, und nicht mehr Beinerhalt oder der klinische Vorteil einer wenig traumatischen Therapie und ihrer Dauerhaftigkeit. Oder um es mit den Worten von Roger Greenhalgh zu sagen: „Patency matters if you have it"!

Müssen wir jetzt umdenken? Sicherlich nicht hinsichtlich der Qualität der Studienendpunkte.

Die in der Gefäßperipherie durchgeführten Studien mit DES [ZilverPTX am Oberschenkel [2] oder Drug Eluting Stents In The Critically Ischemic Lower Leg (DESTINY, [3]) am Unterschenkel] und DEB (Thunder, [4]) belegten statistisch signifikant niedrigere Restenoseraten und TLR. Hinsichtlich der klinischen Endpunkte "clinical benefit", „limb loss“ und ,minor amputation“ oder "severe adverse event" sind die Ergebnisse entweder sehr inhomogen oder zum Nachteil der DET, oder sie wurden gar nicht abgefragt. Die Mortalität in der Thunder-Studie betrug nach 6 Monaten $2 \%$ in der Kontrollgruppe und $4 \%$ in der DEB-Gruppe; die Amputationsrate (Majoramputation) betrug $4 \%$ in der DEB-Gruppe gegenüber $0 \%$ in der Kontrollgruppe. Auch die Baseline-Daten der Patienten weisen auf deutliche Unterschiede hin. In der FemPac-Studie [11] war die Zahl der Komplettverschlüsse der A. femoralis superficialis (AFS) in der Kontrollgruppe um mehr als 50\% höher als in DEB-Gruppe. Eingedenk der Ergebnisse der ViennaAbsolute-Studie von Schillinger et al. [9] haben diese Läsionen ein primär schlechteres Outcome zu erwarten, wenn sie nur mit Ballon-PTA versorgt werden.

Die Diskrepanz zwischen "patency“ und „limb salvage rate“ oder „clinical benefit" zeigt sich in gleicher Weise für die DES-Studien im Unterschenkelsegment; die Offenheitsrate der Stents war signifikant höher, die Beinerhaltungsrate statistisch nicht unterschiedlich (abgesehen von der monozentrischen PaRadise-Studie [6]. 
Es gilt daher, nicht kritiklos die Euphorie zu teilen, sondern die vorliegenden Studien einer kritischen Evaluierung hinsichtlich der Endpunktdefinition und Vergleichbarkeit mit anderen Studien zu unterziehen, wie bereits von Antoniou et al. [1] im Journal of Endovascular Therapy 2013 publiziert.

Folgende Fragen müssen wir stellen:

- Welcher Studienendpunkt hat für den Kliniker die größte Aussagekraft. Ist ein Unterschied von 0,5 mm LLL am Oberschenkel nach 12 Monaten relevant (The Lutonix Paclitaxel-Coated Balloon for the Prevention of Femoropoliteal Restenosis, LEVANT I, [5]) oder muss hier die „dauerhaft erreichbare Verbesserung der Rutherford-Klassifikation“ oder der Beinerhalt als primärer Endpunkt im Protokoll festgelegt sein? Für den Patienten mit kritischer Extremitätenischämie stehen sicherlich der Beinerhalt und die Minoramputationsrate im Vordergrund und nicht die ,primary patency" des Stents [10]

- Sollten die periinterventionelle Komplikationsrate und die Mortalität im Studienverlauf zur Beurteilung als sekundäre Endpunkte mitherangezogen werden, um das Primärrisiko einer Therapie besser zu definieren?

- Ist der Diabetes mellitus weiterhin ein negativ prädiktiver Faktor hinsichtlich „limb loss“ und Mortalität und „primary patency“, oder kann wie in der ZilverPTX ${ }^{\circledR}$-Studie der Drug-eluting-Effekt diesbezüglich ein entscheidender Vorteil sein?

- Lassen die Inhomogenität der Studienendpunkte und Ergebnisse sowie das Fehlen direkter Vergleichsgruppen mit alternativen Strategien eine abschließende Analyse und v. a. eine Schlussfolgerung mit Empfehlung des Verfahrens zu? Liegt nur eine „Endpunkteffektivität" aufgrund des Studiendesigns vor oder besteht aufgrund der klinischen Ergebnisse wirklich die Notwendigkeit, diese Techniken in das Portfolio einer gefäßtherapeutischen Abteilung aufzunehmen?

- Kann man Studienergebnisse mit unterschiedlichen „DE devices“ überhaupt miteinander vergleichen? Die Auswirkungen unterschiedlicher DEB und DES sind nicht komplett gleich. Neben der Vermeidung einer Restenose kann auch die Entwicklung einer lokalen Wandnekrose resultieren [9].

- Sind die höheren Kosten aufgrund der reduzierten Reinterventionsrate gerechtfertigt? Kosteneffektivitätsanalysen in Bezug auf die DET liegen bisher nur ansatzweise und bezogen auf nationale Gegebenheiten vor. Hier soll auf eine französische „Health-economy“Studie in Bezug auf den ZilverPTXStent hingewiesen werden [11].

Die nachfolgenden Beiträge sollen ein Wegweiser durch das Datendickicht sein und die Gesamtheit der Studien für die DEB und DES auf deren wesentliche Aussagen reduzieren. Können die Ergebnisse mit denen anderer Interventionsstudien oder denen von Bypassstudien verglichen werden?

Vielleicht müssen wir einen differenzierten Zugang zu diesen Techniken finden. Wir müssten herausfinden, ob die Gefäßregionen oder die klinischen Bilder, deren Funktion und Verlauf von der Stenose bestimmt werden, eine sichere Indikation für den DEB darstellen, wenn die Restenoserate und der LLL signifikant von der Anwendung der DET profitieren. Dies gilt möglicherweise für Stenosen von atrioventrikulären (AV)-Shunts zur Dialyse oder für den Diabetespatienten mit stenosiertem singulären Unterschenkelgefäß, der aufgrund seiner begleitenden diabetischen Polyneuropathie die belastungsabhängigen Zeichen der kritischen Ischämie nicht spürt und der durch eine präventive Intervention mit dauerhaftem Erfolg vor einer Progression bis hin zum Gewebeverlust bewahrt werden kann.

Die gefäßchirurgische Fachgesellschaft ist hier gefordert, zielführende Studien zu entwickeln und aktiv durchzuführen.

Zweifellos deuten die bisherigen Studienergebnisse darauf hin, dass die DET unsere bisherigen therapeutischen Möglichkeiten erweitern können. Eine liberale Empfehlung hin zum DEB/S und weg von den bisherigen Vorgehensweisen erscheint jedoch in vielerlei Hinsicht verfrüht und nicht evidenzbasiert. Ihr

Dr. J. Teßarek

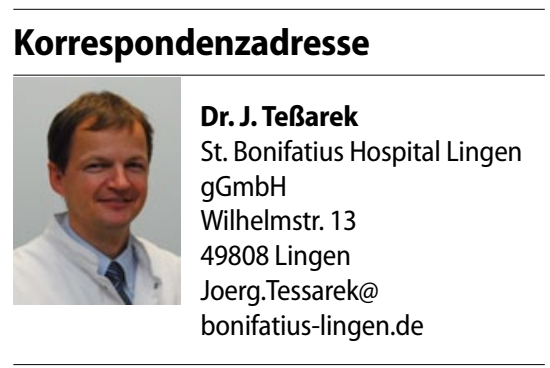

Einhaltung ethischer Richtlinien

Interessenkonflikt. J. Teßarek erhielt einen Research Grant der Fa. Look Medical; er arbeitet mit Abbott zusammen im Brüsseler Crossroads Institute. Mit Cordis zusammen werden endovaskuläre Kurse im ESI in Norderstedt für die DGG abgehalten.

\section{Literatur}

1. Antoniou AA, Chalmers $N$, Kanesalingham $\mathrm{K}$ et al (2013) Metaanalysis of outcomes of endovascular treatment of infrapopliteal occlusive disease with drug eluting stents. J Endovasc Ther 20:131-144

2. Bosiers M, Scheinert D, Peeters P et al (2011) Randomized comparison of everolimus-eluting versus bare-metal-stents in patients with critical limb ischemia and infrapopliteal arterial occlusive disease. J Vasc Surg 55:390-398

3. Dake MD, Scheinert $D$, Tepe $G$ et al; for the Zilver PTX Single-Arm Study Investigators (2011) Nitinol stents with polymer-free paclitaxel coating for lesions in the superficial femoral and popliteal arteries above the knee: twelve-month safety and effectiveness. Results from the Zilver PTX single-arm clinical study. J Endovasc Ther 18:613-623

4. De Cock E, Sapoval M, Julia P et al (2013) A budget impact model for paclitaxel-eluting stent in femoropopliteal disease in france. Cardiovasc Intervent Radiol 36:362-370

5. Duda SH (2012) A review of peripheral DCB clinical trials. Presented on Leipzig Interventional Course (LINC) 2012

6. Feiring AJ, Krahn A, Nelson L et al (2010) Preventing leg amputations in critical limb ischemia with below-the-knee drug-eluting stents. The PaRADISE Trial. J Am Coll Cardiol 55:1580-1589

7. Norgren L, Hiatt WR, Dormandy JA et al (2007) Inter-society consensus for the management of peripheral arterial disease (TASC II). J Vasc Surg 45(Suppl):1-67

8. Rastan A, Tepe G, Krankenberg H et al (2011) Sirolismus-eluting stents versus bare metal stents for treatment of focal lesions in infrapopliteal arteries: a double blind, multi centre, randomized clinical trial. Eur Heart J 32:2274-2281

9. Schillinger M, Sabeti S, Loewe C et al (2006) Balloon angioplasty versus implantation of nitinol stents in the superficial femoral artery. N Engl J Med 354:1879-1888

10. Tepe G, Zeller T, Albrecht T et al (2008) Local delivery of paclitaxel to inhibit restenosis during angioplasty of the leg. N Engl J Med 358:689-99

11. Werk M, Langner S, Reinkensmeier B et al (2008) Inhibition of restenosis in femoropopliteal arteries: paclitaxel-coated versus uncoated balloon: femoral paclitaxel randomized pilot trial. Circulation 118:1358-1365 